\title{
BMC Musculoskeletal Disorders reviewer acknowledgement, 2015
}

James Mockridge

\section{Contributing reviewers}

The editors of BMC Musculoskeletal Disorders would like to thank all our reviewers who have contributed to the journal in Volume 16 (2015).

Ellen Aartun
Canada
H Abdelbary
Canada
Abdellatif Abdelgaied
UK
Ashraf Abdelkafy
Egypt
Adham Abdelrahim
USA
Christina Abdel Shaheed
Australi
Abhishek Abhishek
UK
Mansour Abolghasemian
Iran
Antonio Abramo
Sweden
Jonathan Adachi
Canada
Jo Adams
UK
Adewale Adebajo
UK
Babatunde Adegoke
Nigeria

Ade Fatai Adeniyi

Nigeria

Rintje Agricola

Netherlands

Lamiaa Ahmed

Egypt

Yong Ahn

South Korea

Yasushi Akamatsu

Japan

Hiroshi Akima

Japan

Toru Akiyama

Japan

Esin Aktas Cetin

Turkey

Ali Al Kaissi
Austria

Christoph Albers

Switzerland

John Albright

USA

Luis M. Alegre

Spain

Håkan Alfredson

Sweden
Hamza Alizai

USA

Saad Alsaadi

Australia

Tjarco D.W. Alta

Netherlands

Saad Altaher

Saudi Arabia

Rd Altman

USA

Norio Amizuka

Japan

Juan-Manuel Anaya

Colombia

Amy Anderson

UK

I. Andia

Spain

Dimosthenis Andreou

Germany

Jose Luis Andreu

Spain

Stephen Andrews

Canada

James Andrews

USA 
Ricardo Angeles

Canada

Chiara Angeletti

Italy

Naffis Anjarwalla

UK

Narender Annapureddy

USA

Lieven Annemans

Belgium

Samuel Antwi-Baffour

Ghana

Daisuke Araki

Japan

Nigel Arden

UK

Luca Paolo Ardigò

Italy

Kristina Areskoug Josefsson

Sweden

Martin Aringer

Germany

Terra Arnason

Canada

Toni Arndt

Sweden

Camilla Arvinius

Spain

Elisa Assirelli

Italy

Diego Astur

Brazil

Henry Dushan Edward Atkinson

UK

Halil Atmaca

Turkey

Mukundan Attur

USA

Fabiola Atzeni

Italy

Angelo Gabriele Aulisa

Italy

Iben Axén

Sweden

Mostafa Ayoub

Egypt
Daniel Azevedo

Brazil

Debora Azevedo

Brazil

Fabio Azevedo

Brazil

Jaume Bacardit

UK

Frances Bach

Netherlands

Dawid Baczkowicz

Poland

Carlos Bagley

USA

Clemens Baier

Germany

Daniel Balbachevsky

Brazil

Antoine Baldassari

USA

Alejandro Balsa

Spain

Raveendhara Bannuru

USA

Mary Barbe

USA

Kim Barber Foss

USA

Alexandre Barbosa

Brazil

Thomas Bardin

France

William R. Barfield

USA

Alexej Barg

Switzerland

Antonio Barquet

Uruguay

Helena Barreto Henriksson

Sweden

Dominik Baschera

Switzerland

Caroline Bastiaenen

Netherlands

Marcus Bateman

UK
Christoph Bauer

Switzerland

Catherine Bauge

France

Petra Bäumler

Germany

Murad Bavbek

Turkey

Adam Baxter-Jones

Canada

Anne-Christine Bay-Jensen

Denmark

David Bazett-Jones

USA

Christoph Becher

Germany

Paula Regina Beckenkamp

Australia

Marc Beirer

Germany

Knut Beitzel

Germany

Bulent Bektaser

Turkey

Mary Bell

Canada

Adam Benham

UK

Kathleen Bennett

Ireland

Marco Bergamin

Italy

Mercedes G. Bermudez

Spain

Emilio Besada

Norway

Andrew Beswick

UK

Bryan Beutel

USA

Rahul Bhattacharyya

UK

Harjit Pal Bhattoa

Hungary

Roland Biber

Germany 
Teresa Binkley

USA

Amelia J Birney

USA

Leanne Bisset

Australia

Emmanuel Biver

Switzerland

Jeremy Bland

UK

DJ Blizzard

USA

Dragica Bobinac

Croatia

Christoph Boese

Germany

Richard Bohannon

USA

Megan Bohensky

Australia

Harald Böhm

Germany

David Borenstein

USA

Pieter Koen Bos

Netherlands

Bora Bostan

Turkey

Geoff Bove

USA

Brendan Boyce

USA

Katherine Boyer

USA

Elizabeth Bradley

USA

Laurence Bradley

USA

John Brandsema

USA

Ann Bremander

Sweden

Sharon Brennan-Olsen

Australia

Michael Briggs

UK
Mats Brittberg

Sweden

Alexander Brunner

Switzerland

Paul Bruno

Canada

Adam Bryant

Australia

Stefan Buchmann

Germany

Benjamin Bücking

Germany

Matthew Bucknor

USA

Benjamin Buecking

Germany

Serena Bugatti

Italy

Paul Burgers

Netherlands

Ruben Burgos-Vargas

Mexico

Sigita Burneikiene

USA

Agata Burska

UK

Nathalie Busso

Switzerland

Mario Cabraja

Germany

Henrique Cabrita

Brazil

Libertad Cáceres

Spain

Jerrilyn Cambron

USA

Paul Campbell

UK

Stefano Campi

Italy

Grant Cannon

USA

Patricia Canto

Mexico

Barbara Cappelletto

Italy
Silvia Capuani

Italy

Beatriz Carames Perez

USA

S Carbone

Italy

Mario Cardiel

Mexico

Joshua Carothers

USA

Delesha Carpenter

USA

Andrew Carr

UK

Francesco Carubbi

Italy

Christian Carulli

Italy

Alan Casey

Canada

Matteo Castaldo

Italy

Marco Castori

Italy

Mark Catley

Australia

Luis Catoggio

Argentina

Nicole Cattano

USA

Fulvia Ceccarelli

Italy

Luis Cerezal

Spain

Uphar Chamoli

Australia

BP Chan

Hong Kong

Cliffton Chan

Australia

Kinwei Chan

Taiwan

Eric Chang

USA

Connie Chang

USA 
Eric Chang

USA

Moon Jong Chang

South Korea

Francisco Javier Chaques

Spain

Caroline Chebli

USA

Chun Chen

China

Huajiang Chen

China

Tony Chen

USA

Yen-Jen Chen

Taiwan

Zhaoyu Chen

China

L Cheng

China

Jeffrey Cherian

USA

Louis Cheung

Hong Kong

Wing-Hoi Cheung

Hong Kong

Alessandro Chiarotto

Netherlands

Philip Chilibeck

Canada

Maria Sole Chimenti

Italy

Woojin Cho

USA

Hyon Choi

USA

William Chong

USA

Dean Chou

USA

James Chow

USA

Cara Cipriano

USA

Emma Clark

UK
Bart Clarke

USA

Daniel Cleather

UK

Peter Clegg

UK

Gerald Coakley

UK

Laura Coates

UK

Philip Cohen

USA

Stephen Coleman

USA

Natalie Collins

Australia

James Cone

USA

Richard Conway

Ireland

Chad Cook

USA

Andrew Cooke

Canada

Brooke Coombes

Australia

Oscar J Cordero

Spain

Stephen Cornish

Canada

Pascal Cosette

France

Nigel Cox

UK

Jack Crosbie

Australia

Maria Cubria

USA

Magali Cucchiarini

Germany

Antonio Ignacio Cuesta-Vargas

Spain

Yong Cui

China

Douglas Curran-Everett

USA
Olga Cvijanovic

Croatia

Dorothea Daentzer

Germany

Scott Daffner

USA

Chitra Dahia

USA

Luis Fernando Dahmer Peruchini Brazil

Zoe Dailiana

Greece

Peter Daley

Canada

Michael Dan

Australia

Abhijeet Danve

USA

Amile Dario

Australia

Jose Antonio Da Silva

Portugal

Fereydoun Davatchi

Iran

Aileen Davis

Canada

John Davis

USA

Edward Dawe

UK

Isabel De Salis

UK

Nienke De Vries

Netherlands

Elizabeth Dean

Canada

Benjamin Dean

UK

Carlo Felice De Biase

Italy

Diederik De Cock

Belgium

David Deehan

UK

Massimo De Filippo

Italy 
Jesús Delgado Calle

Spain

Annalisa Del Prete

Italy

Luis Del Rio

Spain

Marco Demange

Brazil

C Deml

Austria

Craig Denegar

USA

Hao Deng

China

Elaine Dennison

UK

Michael Deschenes

USA

Francois Desmeules

Canada

Jacqueline Detert

Germany

James Devocht

USA

Anak Dharmapatni

Australia

Arjun Dhawale

India

Thomas Dienstknecht

Germany

Alberto Diez

Spain

Alexandra Dima

Netherlands

Charles Dinarello

USA

Flavia Di Pietro

Australia

Konstantina Dipla

Greece

Sara Dockrell

Ireland

Dennis Dolny

USA

Julio Domenech

Spain
Davide Maria Donati

Italy

Jian Dong

China

Sergey Dorozhkin

Russian Federation

Aron Downie

Australia

Jeffrey Driban

USA

Georgios Drosos

Greece

Wei Du

China

\section{Arnaud Dubory}

France

Luzi Dubs

Switzerland

Stefan Dudli

Switzerland

Alyssa Dufour

USA

Sampat Dumbre Patil

India

Fiona Duncan

UK

Felix Dyrna Md

USA

Jennifer Earl-Boehm

USA

P. Scott Eastman

USA

Benny Samuel Eathakkattu Antony

Australia

N. Lawrence Edwards

USA

Safak Ekinci

Turkey

Pierre Eleniste

USA

Maha El Gaafary

Egypt

Sherif El Ghazaly

Egypt

Andrew W. Eller

USA
Yasser El Miedany

UK

Sameh Elmorsy

Japan

Mahmoud El-Rosasy

Egypt

Michaela Endres

Germany

Carsten Englert

Germany

Christina Engstrand

Sweden

Antti Eskelinen

Finland

Sean Esmende

USA

Emmanuel Estrella

Philippines

Max Ettinger

Germany

Roni Evans

USA

Nicola Fairhall

Australia

Johannes Fakler

Germany

Miguel Farfán

Colombia

Joshua Farr

USA

Jonathan Farthing

Canada

C Fary

Australia

Marta Favero

Italy

Qi Fei

China

David Feinstein

USA

Reed Ferber

Canada

Robert Ferrari

Canada

Silvano Ferrari

Italy 
Manuela Ferreira

Australia

Jonathan Field

UK

Danilo Fintini

Italy

John Fitzgerald

USA

Martin Fleck

Germany

Tapio Flinkkilä

Finland

Pawel Flont

Poland

Margaret Woon Man Fok

Hong Kong

Samo Fokter

Slovenia

Nancy Forget

Canada

Philipp Forkel

Germany

Nicholas Robert Forsyth

UK

Matteo Fosco

Italy

Calogero Foti

Italy

Andreas Fottner

Germany

Andrew Franklyn-Miller

Australia

Patrick Fransen

Belgium

Michael Freeman

USA

Julien Freitag

Australia

Helen French

Ireland

Simon French

Canada

Arno Frigg

Switzerland

Ts Fu

Taiwan
Eisaku Fujimoto

Japan

Kanji Fukuda

Japan

Naoshi Fukui

Japan

Bridgette Furman

USA

Takefumi Furuya

Japan

André Gächter

Switzerland

Angelo Gaffo

USA

Jacek Gagala

Poland

James Gaida

Australia

Sean Gallagher

USA

Jiri Gallo

Czech Republic

James Galloway

UK

Ek Gamstedt

Sweden

Rajiv Gandhi

Canada

Benjamin Gantenbein

Switzerland

Songtao Gao

China

Yanzheng Gao

China

Juan Garbalosa

USA

Serafin Garcia-Mata

Spain

Pietro Garofalo

Italy

Patel Gayatri

India

Jennifer Geddes-McAlister

Canada

Sebastian Gehmert

Switzerland
Sophie Georgin-Lavialle

France

Bjorn Gerdle

Sweden

Habib Ghasemi

Denmark

Mohammad Ghorbanhoseini USA

Claudia Giacomozzi

Italy

Johannes Giesinger

Netherlands

Bernadette Gillick

USA

Federica Ginanneschi

Italy

Vincenzo Giordano

Brazil

J Girard

France

Melita Giummarra

Australia

Cees Glas

Netherlands

Ronald Glick

USA

Atul Goel

India

Paritosh Gogna

India

Judith Gold

Sweden

Yvonne Golightly

USA

Pedro Augusto Gondim Teixeira France

Rachael Gooberman-Hill

UK

Taco Gosens

Netherlands

Jerzy Gosk

Poland

Richard Gouron

France

Gertraud Gradl

Germany 
Jan Grassmann

Germany

Ed Greenfield

USA

James Greenwood

UK

Phillip Gribble

USA

Xavier Griffin

UK

Tim Griffin

USA

Brunella Grigolo

Italy

Ram Gudavalli

USA

Enrique Guerado

Spain

Paulo Guerra

Brazil

Sasha Gulati

Norway

Jianping Guo

China

Shigong Guo

UK

Zhiling Guo

USA

Prateek Gupta

USA

Carl Haasper

Germany

Kaken Habaxi

China

Sandra Häberle

Germany

Fares S. Haddad

UK

Ellen Merete Hagen

Norway

Yoshiyuki Hakeda

Japan

Alan Hakim

USA

Michiyuki Hakozaki

Japan

Michelle Hall

Australia
Keith Hamel

USA

Sommer Hammoud

USA

Mark Hamrick

USA

Kamel Hamzaoui

Tunisia

Mark Hancock

Australia

Helen Handoll

UK

Matthew Hannon

USA

Tb Hansen

Denmark

Tony Hansson

Sweden

Hideaki Hara

Japan

Timothy Hardingham

UK

Rowan Hardy

Australia

Liora Harel

Israel

Leila Harhaus

Germany

Melissa Harris

Australia

Mark Harrison

Canada

Robert Hartman

USA

Frank Hartmann

Germany

Jan Hartvigsen

Denmark

Yamada Harumoto

Japan

Masahiro Hasegawa

Japan

Robin Haskins

Australia

Gillian Hatfield

Canada

Ellen-Margrethe Hauge

Denmark
Tammy Haut Donahue

USA

Daniël Haverkamp

Netherlands

Eric Hay

France

Daichi Hayashi

USA

David Haynes

Australia

Baorong He

China

Emma Healey

UK

Bryan Heard

Canada

Sajan Hegde

India

Lars Helbig

Germany

Michael Hellman

USA

Yves Henchoz

Switzerland

Dirk Henrich

Germany

A Nders Henricson

Sweden

Yves Henrotin

Belgium

Nicholas Henschke

Germany

Daniel Hensler

Germany

Daniel Hernandez-Vaquero

Spain

Philippe Hernigou

France

Manfred Herold

Austria

Fernando Herrera

USA

Gabriel Herrero-Beaumont Spain

Lise Hestbaek

Denmark

Christoph- E. Heyde

Germany 
John Highton

New Zealand

Jonathan Hill

UK

Stephanie Hinse

Australia

Koji Hiraoka

Japan

Michael Hirschmann

Switzerland

Akihiko Hiyama

Japan

Carmen Ho

Hong Kong

Maik Hoberg

Germany

W Hogge

USA

Lisa Hoglund

USA

Zhang Hong

USA

Thomas Hoogeboom

Netherlands

Davyd R. Hooper

Canada

Gary Hooper

New Zealand

Sven Hoppe

Switzerland

Keisuke Horiuchi

Japan

Ian Horsley

UK

Yuichi Hoshino

Japan

M Hösl

Germany

Toru Hosoi

Japan

Saiyun Hou

USA

Lin-Fen Hsieh

Taiwan

Ru-Lan Hsieh

Taiwan

Jason Hsu

USA
Yi Hua

USA

Da-Geng Huang

China

Hsiaomin Huang

USA

Gail Huber

USA

Erica Huber

Switzerland

Markus Hübscher

Australia

John Hughes

UK

Carel Hulshof

Netherlands

Patricia Hunter

UK

Sheree Hurn

Australia

Kellie Huxel Bliven

USA

Sharon Hyzy

USA

Christoph Ihle

Germany

Thomas Ilchmann

Switzerland

Maria Inacio

Australia

Nevsun Inanc

Turkey

Pier Francesco Indelli

USA

Mm Innmann

Germany

Susumu Inoue

USA

H Inui

Japan

Ulrich Irlenbusch

Germany

Kazunari Ishida

Japan

Muneaki Ishijima

Japan

Yuyu Ishimoto

Japan
Tomotaka Ito

Japan

Norimasa Iwasaki

Japan

Jagnoor Jagnoor

Australia

Babak Jahromi

USA

Sanjeev Jain

India

Nitin Jain

USA

Catherine Jankowski

USA

Hendrik Jansen

Germany

Jacob Jaremko

Canada

Mohamed Jarraya

USA

David Jayne

UK

Ole Kudsk Jensen

Denmark

Tue Secher Jensen

Denmark

Dae-Geun Jeon

South Korea

Jian-Yuan Jiang

China

Lei Jiang

China

Nan Jiang

China

Qing Jiang

China

Kitti Jirarattanaphochai

Thailand

Lara Joaquín

Chile

Beverly Johnson

USA

Bruce Jones

USA

Elena Jones

UK

Andrew Judge

UK 
Shanil Juma

USA

Takahashi Jun

Japan

Jesse Jupiter

USA

Amir RezaKachooei

Iran

Hiroshi Kaji

Japan

George Kalliolias

USA

Sadishkumar Kamalanathan

India

Steven Kamper

Australia

Rita Kandel

Canada

Yuko Kaneko

Japan

Shozo Kanezaki

Japan

James Kang

USA

Bhaveen Kapadia

USA

Christian Kaps

Germany

Saffet Karaca

Turkey

Feyza Karagöz Güzey

Turkey

Joseph Kardouni

USA

Yasmaine Karel

Netherlands

Afshin Karimi

USA

Jaro Karppinen

Finland

Morten Karsdal

Denmark

Philip Kasten

Germany

Susan Kattapuram

USA

Jeffrey Katz

USA
Hoda Kavosi

Iran

Hiroshi Kawaguchi

Japan

Yohei Kawakami

Japan

Seiji Kawano

Japan

Takayuki Kawasaki

Japan

Altaf Ahmad Kawoosa

India

Burcin Kececi

Turkey

Helen Keen

Australia

Joern Kekow

Germany

Joanne Kemp

Australia

Justin Keogh

Australia

J. Christiaan Keurentjes

Netherlands

Tahir Khan

UK

Majed Khraishi

Canada

Piya Kiatisevi

Thailand

Dana Kidder

UK

Jin-Sung Kim

South Korea

Sae Hoon Kim

South Korea

Sung Kyu Kim

South Korea

A Kimura

Japan

Atsushi Kimura

Japan

Yuka Kimura

Japan

T Kirchgesner

Belgium

Chlodwig Kirchhoff

Germany
Serafim Kiriakidis

UK

Andrew Kittelson

USA

Christoph Kittl

Austria

Heidi Klakk

Denmark

Andreas Klatt

Germany

Alison Klika

USA

Matthias C.M. Klotz

Germany

Bas Kluitenberg

Netherlands

M Knobe

Germany

Patrick Knott

USA

Jeffrey Knox

USA

Hiroshi Kobayashi Japan

Takashi Kobayashi

Japan

Hakan Kocaoglu

Turkey

M Koda

Japan

Izaäk Kodde

Netherlands

Cheryl Koehn

Canada

Daisuke Koga

Japan

Hideyuki Koga

Japan

In Jun Koh

South Korea

Zinon Kokkalis

Greece

Werner Kolb

Germany

Elizaveta Kon

Italy

Alice Kongsted

Denmark 


\begin{tabular}{|c|c|c|}
\hline $\begin{array}{l}\text { Kika Konstantinou } \\
\text { UK }\end{array}$ & $\begin{array}{l}\text { Oh-Yun Kwon } \\
\text { South Korea }\end{array}$ & $\begin{array}{l}\text { Andre Leumann } \\
\text { Switzerland }\end{array}$ \\
\hline $\begin{array}{l}\text { Evangelos Kontopantelis } \\
\text { UK }\end{array}$ & $\begin{array}{l}\text { Pauline Siew Mei Lai } \\
\text { Malaysia }\end{array}$ & $\begin{array}{l}\text { Ying Ying Leung } \\
\text { Singapore }\end{array}$ \\
\hline $\begin{array}{l}\text { Kevin Koo } \\
\text { Singapore }\end{array}$ & $\begin{array}{l}\text { Sandra Lakke } \\
\text { Netherlands }\end{array}$ & $\begin{array}{l}\text { Brett Levine } \\
\text { USA }\end{array}$ \\
\hline $\begin{array}{l}\text { Sebastian Kopf } \\
\text { Germany }\end{array}$ & $\begin{array}{l}\text { Polly Lama } \\
\text { USA }\end{array}$ & $\begin{array}{l}\text { Alyn Lewis } \\
\text { UK }\end{array}$ \\
\hline $\begin{array}{l}\text { Panagiotis Korovessis } \\
\text { Greece }\end{array}$ & $\begin{array}{l}\text { José Lamo-Espinosa } \\
\text { Spain }\end{array}$ & $\begin{array}{l}\text { Kirsten Leyland } \\
\text { UK }\end{array}$ \\
\hline $\begin{array}{l}\text { Tanja Kostuj } \\
\text { Germany }\end{array}$ & $\begin{array}{l}\text { Kerstin Landin-Wilhelmsen } \\
\text { Sweden }\end{array}$ & $\begin{array}{l}\text { Ang Li } \\
\text { USA }\end{array}$ \\
\hline $\begin{array}{l}\text { Alpesh Kothari } \\
\text { UK }\end{array}$ & $\begin{array}{l}\text { Jonathan Larkin } \\
\text { USA }\end{array}$ & $\begin{array}{l}\text { Chengliu Li } \\
\text { USA }\end{array}$ \\
\hline $\begin{array}{l}\text { Mark Kotowicz } \\
\text { Australia }\end{array}$ & $\begin{array}{l}\text { Laura Laslett } \\
\text { Australia }\end{array}$ & $\begin{array}{l}\text { Hongshuai Li } \\
\text { USA }\end{array}$ \\
\hline $\begin{array}{l}\text { Eiki Koyama } \\
\text { USA }\end{array}$ & $\begin{array}{l}\text { Richard Lass } \\
\text { Austria }\end{array}$ & $\begin{array}{l}\text { Kuan-Yi Li } \\
\text { Taiwan }\end{array}$ \\
\hline $\begin{array}{l}\text { Leyla Kozaci } \\
\text { Turkey }\end{array}$ & $\begin{array}{l}\text { Aisha Lateef } \\
\text { Singapore }\end{array}$ & $\begin{array}{l}\text { Jing Li } \\
\text { China }\end{array}$ \\
\hline $\begin{array}{l}\text { Agnessa Kozak } \\
\text { Germany }\end{array}$ & $\begin{array}{l}\text { Bruce Latimer } \\
\text { USA }\end{array}$ & $\begin{array}{l}\text { Quan-Zhen Li } \\
\text { USA }\end{array}$ \\
\hline $\begin{array}{l}\text { Franz Kralinger } \\
\text { Austria }\end{array}$ & $\begin{array}{l}\text { Anne Laulund } \\
\text { Denmark }\end{array}$ & $\begin{array}{l}\text { Xudong Li } \\
\text { USA }\end{array}$ \\
\hline $\begin{array}{l}\text { Sandra Kraljevic Pavelic } \\
\text { Croatia }\end{array}$ & $\begin{array}{l}\text { Dana Lawrence } \\
\text { USA }\end{array}$ & $\begin{array}{l}\text { Yong-Qi Li } \\
\text { USA }\end{array}$ \\
\hline $\begin{array}{l}\text { Roman Krawetz } \\
\text { Canada }\end{array}$ & $\begin{array}{l}\text { Andrew Leaver } \\
\text { Australia }\end{array}$ & $\begin{array}{l}\text { Michael Christian Liebensteiner } \\
\text { Austria }\end{array}$ \\
\hline $\begin{array}{l}\text { Wally Krengel } \\
\text { USA }\end{array}$ & $\begin{array}{l}\text { Byung Ho Lee } \\
\text { South Korea }\end{array}$ & $\begin{array}{l}\text { Dennis Liem } \\
\text { Germany }\end{array}$ \\
\hline $\begin{array}{l}\text { Martin Kretzschmar } \\
\text { Switzerland }\end{array}$ & $\begin{array}{l}\text { Dave Lee } \\
\text { Singapore }\end{array}$ & $\begin{array}{l}\text { Seung-Jae Lim } \\
\text { South Korea }\end{array}$ \\
\hline $\begin{array}{l}\text { Morten Tange Kristensen } \\
\text { Denmark }\end{array}$ & $\begin{array}{l}\text { Gun Woo Lee } \\
\text { South Korea }\end{array}$ & $\begin{array}{l}\text { Seung-Jae Lim } \\
\text { South Korea }\end{array}$ \\
\hline $\begin{array}{l}\text { Hollis Krug } \\
\text { USA }\end{array}$ & $\begin{array}{l}\text { Hopin Lee } \\
\text { Australia }\end{array}$ & $\begin{array}{l}\text { Rong Limin } \\
\text { China }\end{array}$ \\
\hline $\begin{array}{l}\text { Jan Kuiper } \\
\text { USA }\end{array}$ & $\begin{array}{l}\text { Jeff Leiter } \\
\text { Canada }\end{array}$ & $\begin{array}{l}\text { Jinn Lin } \\
\text { Taiwan }\end{array}$ \\
\hline $\begin{array}{l}\text { Kenji Kumagai } \\
\text { Japan }\end{array}$ & $\begin{array}{l}\text { Willem Lems } \\
\text { Netherlands }\end{array}$ & $\begin{array}{l}\text { Cheng-Feng Lin } \\
\text { Taiwan }\end{array}$ \\
\hline $\begin{array}{l}\text { Malhar Kumar } \\
\text { India }\end{array}$ & $\begin{array}{l}\text { Andreas Leonidou } \\
\text { UK }\end{array}$ & $\begin{array}{l}\text { Tzu-Hua Lin } \\
\text { USA }\end{array}$ \\
\hline $\begin{array}{l}\text { Yogesh Kumar } \\
\text { USA }\end{array}$ & $\begin{array}{l}\text { Panagiotis Lepetsos } \\
\text { Greece }\end{array}$ & $\begin{array}{l}\text { Liow Lincoln } \\
\text { Singapore }\end{array}$ \\
\hline $\begin{array}{l}\text { Steve Kurtz } \\
\text { USA }\end{array}$ & $\begin{array}{l}\text { Lindsey Lepley } \\
\text { USA }\end{array}$ & $\begin{array}{l}\text { Emily Lindley } \\
\text { USA }\end{array}$ \\
\hline $\begin{array}{l}\text { Linda Kwakkenbos } \\
\text { Canada }\end{array}$ & $\begin{array}{l}\text { Gemma Lepri } \\
\text { Italy }\end{array}$ & $\begin{array}{l}\text { Rene Lindstrøm } \\
\text { Denmark }\end{array}$ \\
\hline
\end{tabular}


David R Lionberger

USA

Sabine Lippacher

Germany

Sebastian Lippross

Germany

Gina Lisignoli

Italy

Chris Littlewood

UK

Jiaming Liu

China

Jun Liu

China

Junting Liu

China

Liehua Liu

China

R Liu

USA

Shaoyu Liu

China

Lisa Lix

Canada

Margery Lockard

USA

Alayna Loiselle

USA

Gloria Lopez

Spain

Mattia Loppini

Italy

JK Lord

USA

John Loughlin

UK

Quinette Louw

South Africa

Anna Lowe

UK

Linchao Lu

USA

Xuhua Lu

China

Yu Lu

China

Gregory Lubiani

USA
Brittney Luc

USA

Juan Luciano

Spain

Nada Lukkahatai

USA

Teija Lund

Finland

Jiaquan Luo

China

Hannu Luomajoki

Switzerland

Alejandro Luque-Suarez

Spain

Shai Luria

Israel

Frank Luyten

Belgium

Shaw-Ruey Lyu

Taiwan

Luciana Macedo

Canada

Gustavo Machado

Australia

George Macheras

Greece

Martin Mackey

Australia

Fiona MacKichan

UK

Shingo Maeda

Japan

Nicola Maffulli

UK

Costis Maganaris

UK

Asmaa Mahmoud Ali Moustafa

Egypt

Edyta Majorczyk

Poland

Sharmila Majumdar

USA

Kin Cheung Mak

Hong Kong

Charles Malemud

USA

Rajesh Malhotra

India
Theodore Malinin

USA

Krishnamurthy Saraswathi Manjunath

India

Sithombo Maqungo

South Africa

Meir Marmor

USA

Antonio Marmotti

Italy

Hubert Marotte

France

Elisa Marques

Portugal

Frank Martetschläger

Germany

Janne Martikainen

Finland

Nicolo Martinelli

Italy

Daniel Martinez-Laguna

Spain

M Martin-Ferrero

Spain

Youssef Masharawi

Israel

Stefano Masiero

Italy

Aidin Masoudi

USA

Tahir Masud

UK

Yuichiro Matsui

Japan

Glenn Matsushima

USA

Takehiko Matsushita

Japan

Tokio Matsuzaki

Japan

Stefano Mattioli

Italy

G Matziolis

Germany

Andreas Mavrogenis

Greece

Paolo Mazzola

Italy 
Chidozie Mbada

Nigeria

Jenny McConnell

Australia

Daniel McCulloch

Australia

Catriona McDaid

UK

Suzanne McDonough

USA

Meghan McGee-Lawrence

USA

Tracy McGregor

USA

Neil McHugh

UK

C. Wayne McIlwraith

USA

Aideen McInerney-Leo

Australia

Eileen McNeely

USA

Amy McNulty

USA

Emily McWalter

USA

Daniel McWilliams

UK

Damian Medici

USA

Geert Meermans

Belgium

Christian Meier

Switzerland

Marrigje Meijer

Netherlands

Riccardo Meliconi

Italy

Stephen Mellon

UK

Ehud Mendel

USA

Christopher Mendias

USA

Matthew Menon

Canada

Demissew Mern

Austria
Addisu Mesfin

USA

Karen Messing

Canada

James Michelson

USA

Toshimi Michigami

Japan

Michael Mienaltowski

USA

Paola Migliorini

Italy

Salvatore Minnella

Italy

Frederico Miranda

Brazil

Smarak Mishra

UK

Thomas Mittlmeier

Germany

Junichi Miyake

Japan

Takeshi Miyamoto

Japan

Ali Mobasheri

UK

Maarten Moen

Netherlands

Omar Mohamed

Germany

Mohamed Mohi Eldin

Egypt

Rahim Moineddin

Canada

Ingrid Moller

Spain

Halima Moncrieffe

USA

Robert Moots

UK

B Moradi

Germany

Jaime Morales

Spain

Kanji Mori

Japan

Fintan Moriarty

Switzerland
Suzanne Morin

Canada

Akio Morinobu

Japan

Toru Moro

Japan

Rohan Morris

UK

Seyed Javad Mousavi

Australia

Sara Muller

UK

Marcus Mumme

Switzerland

Shigeyuki Muraki

Japan

Tsuyoshi Murase

Japan

George Murrell

Australia

Harun Mutlu

Turkey

Es Mwaka

Uganda

Corrie Myburgh

Denmark

Keiji Nagata

Japan

Istvan Nagy

UK

Bindu Nair

Canada

Yusuke Nakagawa

Japan

Atsuo Nakamae

Japan

Naoki Nakano

Japan

Tomoyuki Nakasa

Japan

Arvind Nana

USA

Kutty Selva Nandakumar

Sweden

Michael Nasr

USA

Nagwa Nassar

Egypt 
Jose Luis Navarro-Espigares

Spain

Sangeetha Naveen

Malaysia

Justine Naylor

Australia

Ara Nazarian

USA

Mwidimi Ndosi

UK

Subas Neupane

Finland

D Newell

UK

Johnathan Ng

USA

Leo Ng

Australia

Leslie Nicholson

Australia

Andreas Niemeier

Germany

Thomas Niethammer

Germany

Pieter Nieuwenhuijzen

Netherlands

Svetla Nikolova

Bulgaria

Jun Nishida

Japan

Yuichiro Nishizawa

USA

Daniele Noel

France

Marcello Nogueira-Barbosa

Brazil

JH Noh South

Korea

Peter Norman

UK

Andrej Nowakowski

Switzerland

Georgia Ntani

UK

Mary Nugent

Ireland

Michael Nunns

UK
Alison Oates

Canada

Neil O'Connell

UK

Pat O'Connor

USA

David O'Gorman

Canada

Britt Elin Øiestad

Norway

Mary O'Keeffe

Ireland

Francesco Oliva

Italy

Rene Olivares-Navarrete

USA

Chris Oliver

UK

Gunnar Olsson

Sweden

Go Omori

Japan

Patrick Omoumi

Switzerland

Raymond Oppong

UK

Ana-Maria Orbai

USA

Viveca Östberg

Sweden

Monika Ostensen

Norway

Georg Osterhoff

Switzerland

Kieran O'Sullivan

Ireland

Leonard O'Sullivan

Ireland

Takemi Otsuki

Japan

Merry Oursler

USA

Simon Øverland

Norway

Levent Ozcakar

Turkey

Guven Ozkaya

Turkey
Keiichi Ozono

Japan

Yusuf Öztürkmen

Turkey

Patrick Pabian

USA

Verity Pacey

Australia

Jukka Pajarinen

USA

Paolo Paladini

Italy

Shea Palmer

UK

Thorvaldur Palsson

Denmark

Wen Pan

USA

Danilo Pani

Italy

Ranajit Panigrahi India

Evangelos Pappas

Australia

John Pappas

USA

Eric Parent

Canada

Jang Won Park

South Korea

Jin KyunPark

South Korea

Won Sang Park

South Korea

Martyn Parker

UK

Sharon Parry

Australia

Zoe Paskins

UK

Debabrata Patra

USA

Jochen Paul

Germany

Elena Pavlova

UK

Imre Pavo

Austria 


Mark Pearson
UK
Ashley Pedler
Australia
Nancye May Peel
Australia
Fuxing Pei
China

\section{Zhang Peixun}

China

Leonardo Pellicciari

Italy

Baogan Peng

China

Erika Penz

Canada

Anneli Peolsson

Sweden

Melanie Pepin

USA

Beatriz Pérez-Zafrilla

Spain

Harris Perlman

USA

Yves-Marie Pers

France

Ákos Pethes

Hungary

Susan Picavet

Netherlands

Motta Pierorazio

Italy

Patrícia Pinto

Portugal

Rafael Pinto

Australia

Spiros Pneumaticos

Greece

Denis Poddubnyy

Germany

Jonas Pogorzelski

Germany

Brian Poole

USA

Hadi Poormoghim

Iran

Janet Pope

Canada
Mark Porcheret

UK

Alexey Porollo

USA

Fereshteh Pourkazemi

Australia

James Prior

UK

Ashleigh Prowse

Australia

Thomas Pufe

Germany

Paul Purdue

USA

Zhao Qing Hua

China

Gerald Quan

Australia

Danuta Radzioch

Canada

Stefan Rahm

Switzerland

Muhammad Farooq Rai

USA

Yolande F. M. Ramos

Netherlands

Francesco Ranuccio

Italy

Lasse Enkeboelle Rasmussen

Denmark

Eva Rasmussen-Barr

Sweden

Akhilesh Rathi

India

Michael Skovdal Rathleff

Denmark

Trishna Rathod

UK

Frank Rauch

Canada

Jennifer Read

UK

Michael Reade

Australia

Trudy Rebbeck

Australia

Sakamuri Reddy

USA
Martine Regenboog

Netherlands

Thomas Reichel

Germany

Duncan Reid

New Zealand

Monique Reijnierse

Netherlands

Olav Reikerås

Norway

Michael Reiman

USA

Jens Reimann

Germany

Dajiang Ren

China

Xiang Ren

China

Babst Reto

Switzerland

Jose A. Riancho

Spain

Marcelo Riberto

Brazil

Jim Richards

UK

Robin Richards

USA

James B Richardson

UK

Julie Richardson

Canada

Jane Richardson

UK

Jochen Ringe

Germany

Bernhard Rintelen

Austria

Fernando Rivadeneira

Netherlands

Shawn Robbins

Canada

Timothy Roberts

USA

Philip Robinson

Australia

Rita Rocha

Portugal 
Scott Rodeo

USA

Götz Röderer

Germany

Vitor Rodrigues

Portugal

Sattaya Rojanasthien

Thailand

Tobias Romeyke

Austria

Keith Rose

USA

Joseph Rosen

USA

Dieter Rosenbaum

Germany

Ann Rosenthal

USA

Barbara Rossi

Italy

Roberto Rossi

Italy

Graciela Rovner

Sweden

Flynn Rowan

USA

Paula Rozenfeld

Argentina

Dike Ruan

China

L. Ruggiero

Italy

Cormac Ryan

UK

Joachim Rychly

Germany

Luigi Sabatini

Italy

Ahmed Sadek

Egypt

Patrick Sadoghi

Austria

Surachai Sae-Jung

Thailand

Jayaprakash Sahoo

India

Kulvinder Singh Saini

Saudi Arabia
Taku Saito

Japan

Masayuki Saka

Japan

Takashi Sakai

Japan

Akio Sakamoto

Japan

Km Salem

UK

Donald Salter

UK

John Salvo

USA

Afshin Samani

Denmark

Nemandra Sandiford

UK

Gunther H. Sandmann

Germany

Bruno Saragiotto

Australia

Joseph Sarver

USA

Vishal Sarwahi

USA

Hiroshi Sasaki

USA

Ben Saunders

UK

Judith Sautner

Austria

Olga Savvidou

Greece

Tetsuji Sawada

Japan

Frederieke Schaafsma

Australia

Ellen Scherl

USA

Philip Scheurer

Netherlands

Aaron Schindeler

Australia

Ulf Jens Schlegel

Germany

Anneliese Schleyer

USA
Benedikt Schliemann

Germany

Tanja Schmidt

Germany

Tannin Schmidt

Canada

J Schmolders

Germany

Michael Schnabel

Germany

Michael Schneider

USA

Arndt P. Schulz

Germany

Gundula Schulze-Tanzil

Germany

Philipp Schwabe

Germany

Cedric Schwartz

Belgium

Edward Schwarz

USA

Ran Schwarzkopf

USA

Richard Schwend

USA

Jason Scibek

USA

John Scolaro

USA

David Scott

UK

Whitney Scott

UK

Anna Scotto D'Abusco

Italy

Mamdouh Sedhom

Australia

Leyla Sedighipour

USA

Miho Sekiguchi

Japan

Jonathan Sembrano

USA

Emily Seto

Canada

F Sevelda

Austria 


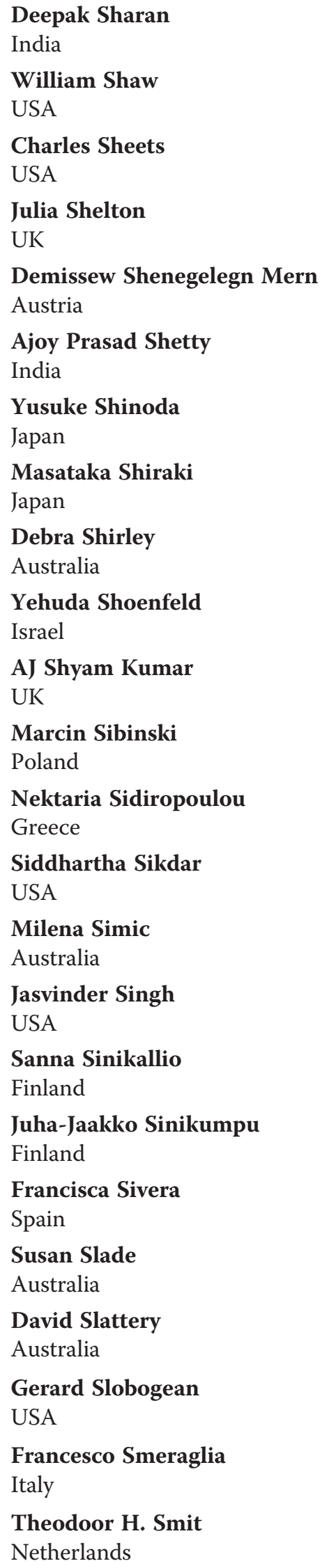

\begin{tabular}{|c|c|}
\hline $\begin{array}{l}\text { Jo Smith } \\
\text { UK }\end{array}$ & $\begin{array}{l}\text { Sebastian Suarez } \\
\text { USA }\end{array}$ \\
\hline $\begin{array}{l}\text { Lachlan Smith } \\
\text { USA }\end{array}$ & $\begin{array}{l}\text { A Sud } \\
\text { India }\end{array}$ \\
\hline $\begin{array}{l}\text { Tuulikki Sokka } \\
\text { Finland }\end{array}$ & $\begin{array}{l}\text { Tatsuo Suda } \\
\text { Japan }\end{array}$ \\
\hline $\begin{array}{l}\text { Leonid Solomin } \\
\text { Russian Federation }\end{array}$ & $\begin{array}{l}\text { Akihiro Sudo } \\
\text { Japan }\end{array}$ \\
\hline $\begin{array}{l}\text { Matthijs Somford } \\
\text { Netherlands }\end{array}$ & $\begin{array}{l}\text { Ruifang Sui } \\
\text { China }\end{array}$ \\
\hline $\begin{array}{l}\text { Enrique R. Soriano } \\
\text { Argentina }\end{array}$ & $\begin{array}{l}\text { Walton Sumner } \\
\text { USA }\end{array}$ \\
\hline $\begin{array}{l}\text { Miguel Sousa } \\
\text { Portugal }\end{array}$ & $\begin{array}{l}\text { Yu Sun } \\
\text { China }\end{array}$ \\
\hline $\begin{array}{l}\text { Richard Souza } \\
\text { USA }\end{array}$ & $\begin{array}{l}\text { Björn Sundström } \\
\text { Sweden }\end{array}$ \\
\hline $\begin{array}{l}\text { Dusko Spasovski } \\
\text { Serbia }\end{array}$ & $\begin{array}{l}\text { Wen-Hsu Sung } \\
\text { Taiwan }\end{array}$ \\
\hline $\begin{array}{l}\text { Mauro Spina } \\
\text { Italy }\end{array}$ & $\begin{array}{l}\text { Akari Suzuki } \\
\text { Japan }\end{array}$ \\
\hline $\begin{array}{l}\text { Christian Spross } \\
\text { Switzerland }\end{array}$ & $\begin{array}{l}\text { Noboru Suzuki } \\
\text { Japan }\end{array}$ \\
\hline $\begin{array}{l}\text { Sander Spruijt } \\
\text { Netherlands }\end{array}$ & $\begin{array}{l}\text { Tomoyuki Suzuki } \\
\text { Japan }\end{array}$ \\
\hline $\begin{array}{l}\text { J. Bart Staal } \\
\text { Netherlands }\end{array}$ & $\begin{array}{l}\text { Michael Swain } \\
\text { Australia }\end{array}$ \\
\hline $\begin{array}{l}\text { D. Stacey } \\
\text { Canada }\end{array}$ & $\begin{array}{l}\text { Ganesh Swamy } \\
\text { Canada }\end{array}$ \\
\hline $\begin{array}{l}\text { Rebecca Stack } \\
\text { UK }\end{array}$ & $\begin{array}{l}\text { Grazyna Sypniewska } \\
\text { Poland }\end{array}$ \\
\hline $\begin{array}{l}\text { Daniel Steffens } \\
\text { Australia }\end{array}$ & $\begin{array}{l}\text { Uta Syrbe } \\
\text { Germany }\end{array}$ \\
\hline $\begin{array}{l}\text { Ely Steinberg } \\
\text { Israel }\end{array}$ & $\begin{array}{l}\text { Pawel Szulc } \\
\text { France }\end{array}$ \\
\hline $\begin{array}{l}\text { Arnd Steinbrueck } \\
\text { Germany }\end{array}$ & $\begin{array}{l}\text { Sofi Tagesson } \\
\text { Sweden }\end{array}$ \\
\hline $\begin{array}{l}\text { Dirk Stengel } \\
\text { Germany }\end{array}$ & $\begin{array}{l}\text { Mohammad Taghipour Darzi } \\
\text { Iran }\end{array}$ \\
\hline $\begin{array}{l}\text { Joanna Stephen } \\
\text { UK }\end{array}$ & $\begin{array}{l}\text { Yoo Tai June } \\
\text { USA }\end{array}$ \\
\hline $\begin{array}{l}\text { Matthew Stevens } \\
\text { Australia }\end{array}$ & $\begin{array}{l}\text { Toshiaki Takahashi } \\
\text { Japan }\end{array}$ \\
\hline $\begin{array}{l}\text { Stefan Stich } \\
\text { Germany }\end{array}$ & $\begin{array}{l}\text { Naoyuki Takahashi } \\
\text { Japan }\end{array}$ \\
\hline $\begin{array}{l}\text { Mette Stochkendahl } \\
\text { Denmark }\end{array}$ & $\begin{array}{l}\text { Koji Takayama } \\
\text { Japan }\end{array}$ \\
\hline $\begin{array}{l}\text { Klaus Strobel } \\
\text { Switzerland }\end{array}$ & $\begin{array}{l}\text { Yuji Takazawa } \\
\text { Japan }\end{array}$ \\
\hline
\end{tabular}


Wen-Hua Tan

China

Nobuhiro Tanaka

Japan

Veena Taneja

USA

Chih-Hsin Tang

Taiwan

Noboru Taniguchi

Japan

Sarah Tansley

UK

Ekamol Tantisattamo

USA

Anne-Kathrin Tausche

Germany

Rod Taylor

UK

Regina Taylor-Gjevre

Canada

Andrew Teichtahl

Australia

René Ten Broeke

Netherlands

Masafumi Terada

USA

Masatoshi Teraguchi

Japan

Takeshi Teratani

Japan

Enrique Adrian Testa

Switzerland

Teun Teunis

Netherlands

Loukas Thanos

Greece

Jean-Claude Theis New

Zealand

Brian Theodore

USA

Achilleas Thoma

Canada

Gethin Thomas

Australia

Ellen Thomas

UK

Martin Thomas

UK
Jeffrey Thomson

USA

Andrew Thoreson

USA

Stephanie Tien-A-Looi

USA

Mark Timmons

USA

Thomas Tischer

Germany

Derrick Todd

USA

Stefan Toegel

Austria

Andreas Toepfer

Germany

Harukazu Tohyama

Japan

Christy Tomkins-Lane

Canada

Peijian Tong

China

Daniel Tonge

UK

Francine Toye

UK

Ismail Remzi Tozun

Turkey

Marco Traballesi

Italy

Adrian Traeger

Australia

Vincent Traynelis

USA

Lea Trela-Larsen

UK

Ilaria Tresoldi

Italy

Luisa Trombi

Italy

Kimberlee Trudeau

USA

Aleksandra Truszczynska

Poland

Tsung-Yuan Tsai

USA

Elena Tsourdi

Germany
Sachiyuki Tsukada

Japan

Marcin Tyrakowski

Poland

Jan Ulfberg

Sweden

J Urrutia

Chile

Werner Vach

Germany

Guus Van Den Akker

Netherlands

Bart Van Den Bemt

Netherlands

Peter Van Den Berg

Netherlands

Paul Van Den Dolder

Australia

Cornelia Van Den Ende

Netherlands

Josien Van Den Noort

Netherlands

Walter Van Der Weegen

Netherlands

Danielle Van Der Windt

UK

Peter Van Lent

Netherlands

Gerjo Van Osch

Netherlands

Ottar Vasseljen

Norway

Martti Vastamäki

Finland

Patrick Vavken

USA

Arianne Verhagen

Netherlands

John Verhoef

Netherlands

Howard Vernon

Canada

Francesca Veronesi

Italy

Martin L Verra

Switzerland

Suzanne Verstappen

UK 


\begin{tabular}{|c|}
\hline $\begin{array}{l}\text { E Vialle } \\
\text { Brazil }\end{array}$ \\
\hline $\begin{array}{l}\text { Esther Vicente } \\
\text { Spain }\end{array}$ \\
\hline $\begin{array}{l}\text { Tapio Videman } \\
\text { Canada }\end{array}$ \\
\hline $\begin{array}{l}\text { Edgar Vieira } \\
\text { USA }\end{array}$ \\
\hline $\begin{array}{l}\text { Davide Viggiano } \\
\text { Italy }\end{array}$ \\
\hline $\begin{array}{l}\text { Juan Villa-Camacho } \\
\text { USA }\end{array}$ \\
\hline $\begin{array}{l}\text { Rob Vining } \\
\text { USA }\end{array}$ \\
\hline $\begin{array}{l}\text { Marianna Vlychou } \\
\text { Greece }\end{array}$ \\
\hline $\begin{array}{l}\text { Nam Vo } \\
\text { USA }\end{array}$ \\
\hline $\begin{array}{l}\text { Stephan Vogt } \\
\text { Germany }\end{array}$ \\
\hline $\begin{array}{l}\text { Mauro Volpi } \\
\text { Brazil }\end{array}$ \\
\hline $\begin{array}{l}\text { Philipp Von Roth } \\
\text { Germany }\end{array}$ \\
\hline $\begin{array}{l}\text { Christian Von Rüden } \\
\text { Germany }\end{array}$ \\
\hline $\begin{array}{l}\text { James Vosseller } \\
\text { USA }\end{array}$ \\
\hline $\begin{array}{l}\text { Alessandra Vultaggio } \\
\text { Italy }\end{array}$ \\
\hline $\begin{array}{l}\text { Raimund Wagener } \\
\text { Germany }\end{array}$ \\
\hline $\begin{array}{l}\text { Monique Margaretha } \\
\text { Jozefa Walenkamp } \\
\text { Netherlands }\end{array}$ \\
\hline $\begin{array}{l}\text { William Walsh } \\
\text { Australia }\end{array}$ \\
\hline $\begin{array}{l}\text { Nicole Walsh } \\
\text { Australia }\end{array}$ \\
\hline $\begin{array}{l}\text { Edward Wang } \\
\text { Philippines }\end{array}$ \\
\hline $\begin{array}{l}\text { James Wang } \\
\text { USA }\end{array}$ \\
\hline $\begin{array}{l}\text { Jann-Tay Wang } \\
\text { Taiwan }\end{array}$ \\
\hline $\begin{array}{l}\text { Jing Wang } \\
\text { USA }\end{array}$ \\
\hline
\end{tabular}

\begin{tabular}{|c|c|}
\hline $\begin{array}{l}\text { Shaobai Wang } \\
\text { USA }\end{array}$ & $\begin{array}{l}\text { Karl-Heinz Widmer } \\
\text { Switzerland }\end{array}$ \\
\hline $\begin{array}{l}\text { Yuanyuan Wang } \\
\text { Australia }\end{array}$ & $\begin{array}{l}\text { Susan Wilbanks } \\
\text { UK }\end{array}$ \\
\hline $\begin{array}{l}\text { Yum-Tao Wang } \\
\text { China }\end{array}$ & $\begin{array}{l}\text { Amanda Williams } \\
\text { UK }\end{array}$ \\
\hline $\begin{array}{l}\text { Michael Ward } \\
\text { USA }\end{array}$ & $\begin{array}{l}\text { Christopher Williams } \\
\text { Australia }\end{array}$ \\
\hline $\begin{array}{l}\text { Stuart Warden } \\
\text { USA }\end{array}$ & $\begin{array}{l}\text { Valerie Williams } \\
\text { USA }\end{array}$ \\
\hline $\begin{array}{l}\text { Fiona Watt } \\
\text { UK }\end{array}$ & $\begin{array}{l}\text { Tobias Winkler } \\
\text { Germany }\end{array}$ \\
\hline $\begin{array}{l}\text { Adam Watts } \\
\text { UK }\end{array}$ & $\begin{array}{l}\text { John Winslow } \\
\text { USA }\end{array}$ \\
\hline $\begin{array}{l}\text { M Weber } \\
\text { Germany }\end{array}$ & $\begin{array}{l}\text { Patarawan Woratanarat } \\
\text { Thailand }\end{array}$ \\
\hline $\begin{array}{l}\text { Fiona Webster } \\
\text { Canada }\end{array}$ & $\begin{array}{l}\text { Stuart Wright } \\
\text { UK }\end{array}$ \\
\hline $\begin{array}{l}\text { Niels Wedderkopp } \\
\text { Denmark }\end{array}$ & $\begin{array}{l}\text { Jau-Ching Wu } \\
\text { Taiwan }\end{array}$ \\
\hline $\begin{array}{l}\text { Kilian Wegmann } \\
\text { Germany }\end{array}$ & $\begin{array}{l}\text { Guoming Xie } \\
\text { China }\end{array}$ \\
\hline $\begin{array}{l}\text { Lei Wei } \\
\text { USA }\end{array}$ & $\begin{array}{l}\text { Jiake Xu } \\
\text { Australia }\end{array}$ \\
\hline $\begin{array}{l}\text { Conrad Weihl } \\
\text { USA }\end{array}$ & $\begin{array}{l}\text { Weidong Xu } \\
\text { China }\end{array}$ \\
\hline $\begin{array}{l}\text { Yoram Weil } \\
\text { Israel }\end{array}$ & $\begin{array}{l}\text { Yue Xuan } \\
\text { USA }\end{array}$ \\
\hline $\begin{array}{l}\text { Andre Weimann } \\
\text { Germany }\end{array}$ & $\begin{array}{l}\text { Meilang Xue } \\
\text { Australia }\end{array}$ \\
\hline $\begin{array}{l}\text { Harrie Weinans } \\
\text { Netherlands }\end{array}$ & $\begin{array}{l}\text { Ryo Yamada } \\
\text { Japan }\end{array}$ \\
\hline $\begin{array}{l}\text { Tomas Weitoft } \\
\text { Sweden }\end{array}$ & $\begin{array}{l}\text { Hisashi Yamanaka } \\
\text { Japan }\end{array}$ \\
\hline $\begin{array}{l}\text { Megan Weivoda } \\
\text { USA }\end{array}$ & $\begin{array}{l}\text { RJ Yan } \\
\text { China }\end{array}$ \\
\hline $\begin{array}{l}\text { Chunyi Wen } \\
\text { Hong Kong }\end{array}$ & $\begin{array}{l}\text { Huilin Yang } \\
\text { China }\end{array}$ \\
\hline $\begin{array}{l}\text { Maria M. Wertli } \\
\text { Switzerland }\end{array}$ & $\begin{array}{l}\text { Yun-Feng Yang } \\
\text { China }\end{array}$ \\
\hline $\begin{array}{l}\text { Michael Whitehouse } \\
\text { UK }\end{array}$ & $\begin{array}{l}\text { Qiuming Yao } \\
\text { USA }\end{array}$ \\
\hline $\begin{array}{l}\text { David Whitehurst } \\
\text { Canada }\end{array}$ & $\begin{array}{l}\text { Hisataka Yasuda } \\
\text { Japan }\end{array}$ \\
\hline $\begin{array}{l}\text { Rod Whiteley } \\
\text { Qatar }\end{array}$ & $\begin{array}{l}\text { Kensuke Yasumura } \\
\text { Japan }\end{array}$ \\
\hline $\begin{array}{l}\text { Harald Widhalm } \\
\text { Austria }\end{array}$ & $\begin{array}{l}\text { Metin Yavuz } \\
\text { USA }\end{array}$ \\
\hline
\end{tabular}




Xiaodong Yi
China
M Yoshida
Japan
Hiroyuki Yoshihara
USA
Toshitaka Yoshii
Japan
Ichiro Yoshimura
Japan
Hiroyuki Yoshitomi
Japan
Md Yuzaiful Md Yusof
UK
Xiuchun Yu
China
Hua Yu
USA
W Yuan
China
Kazuo Yudoh
Japan

Liu Yueju

China

I Yugué

Japan

Andreas Zafeiridis

Greece

Fabio Zaina

Italy

Ivan Zderic

USA

Jian Zhang

USA

Jun Zhang

China

Kairui Zhang

China

Qiang Zhang

China

Xiaofan Zhang

USA

Xiaojian Zhang

China
Ying Zhang

China

Fengdong Zhao

China

Yong Zhao

China

Liying Zheng

USA

Fang Zhenhua

China

Yongjin Zhou

China

Linuo Zhou

China

Yuan Zhu

China

Ruofu Zhu

China

Yi Zhu

USA 